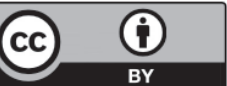

https://creativecommons.org/licenses/by/4.0/

\title{
ANÁLISIS DEL MANTENIMIENTO CORRECTIVO EN UN SISTEMA PRODUCTIVO DE ETIQUETAS TERMO-ENCOGIBLES, POR MEDIO DE CADENAS DE MARKOV
}

\author{
Analysis for corrective maintenance in shrink \\ label productive system, through Markov Chains
}

LAURA ANGÉLICA MEJÍA OSPINA*, DIANA CAROLINA URBANO PASTUSANO**, JORGE RASHID PÉREZ PRADA***

Recibido: 12 de junio de 2016. Aceptado: 23 de junio de 2016

DOI:http://dx.doi.org/10.21017/rimci.2016.v3.n6.a11

\begin{abstract}
Resumen
La aplicación de Cadenas de Markov surte especial importancia a la hora de controlar procesos como de gestión de inventarios en el campo de la Ingeniería Industrial. Esta investigación ilustra la aplicación de esta técnica de lnvestigación de Operaciones, con el fin de analizar el comportamiento del mantenimiento correctivo alrededor de un sistema productivo que se dedica a la fabricación de etiquetas termo-encogibles. Con el estudio de las probabilidades de estado estable del sistema, y el análisis de los tiempos medios de recurrencia y de primer paso, se evidenció que la política de inventario actual para estos repuestos no suple la demanda para los fallos de estas bombas, y así se pudo determinar una propuesta para replantear esta política.
\end{abstract}

Palabras clave: mantenimiento correctivo, proceso markoviano, gestión de inventarios.

\begin{abstract}
Markov Chain applications are important to make sure of inventory management and control, since Industrial Engineering field. This research shows an Operation Research technique that can be applied to analyze corrective maintenance behavior of a productive system, which focuses on making shrink label. With study of steady-state probabilities System and analysis of the average time of recurrence and first step it was evident that the current inventory policy for these parts not adjust the demand for failures, and it was determined a proposal to rethink this policy.
\end{abstract}

Keywords: corrective mantenaince, markovian process, inventory management.

\section{INTRODUCCIÓN}

Se ilustra un análisis por medio de la técnica Cadenas de Markov, para estudiar el comportamiento del mantenimiento correctivo presentado en una lí- nea de producción de etiquetas termo-encogibles, para una empresa de plásticos en la ciudad de Cali.

Se presenta una alta frecuencia de mantenimientos correctivos por desgaste o daños de piezas en

\footnotetext{
* Ingeniera Industrial y Msc. en Investigación Operativa y Estadística. Actualmente docente del departamento de Operaciones y Sistemas de la Universidad Autónoma de Occidente. Su experiencia incluye procesos de consultoría empresarial, docencia, y desarrollo de capacitaciones alrededor del uso de la lúdica como herramienta pedagógica en el proceso de enseñanza-aprendizaje de Ingeniería Industrial y carreras afines. Correo electrónico: lamejia@uao.edu.co

** Ingeniera Industrial en formación. Octavo semestre. Universidad Autónoma de Occidente. Correo electrónicokarodianau@hotmail.com ***Ingeniero Industrial en formación. Octavo semestre. Universidad Autónoma de Occidente. Correo electrónicojprashid_05@hotmail.com
} 
las bombas peristálticas, con datos y situaciones actuales de tal manera que se identifiquen los componentes más críticos y evaluar el planteamiento de las posibles soluciones con el objeto de no elevar el costo de mantener estas bombas y conocer el stock de repuestos adecuados, para solventar los cambios sin demorar la reparación.

Las bombas peristálticas son un equipo de apoyo importante para el proceso de impresión de las etiquetas termo-encogibles, ya que estás bombean las tintas logrando que no se contaminen con el ambiente, también tienden a sufrir desgaste en sus partes mecánicas y eléctricas por la operación constante y la contaminación de la misma con el fluido que bombea.

Los tiempos para cambio de repuestos están entre 20 a 30 min desde que se recibe la orden de trabajo hasta se entrega el equipo funcionado al operario, teniendo en cuenta que la máquina impresora de etiquetas (SPM) debe contar con 7 bombas en cada una de sus 7 unidades, y que no hay bombas de repuesto y que el no tener una bomba disponible, la unidad de la bomba que falle queda parada, lo que quiere decir que la impresora SMP solo funcionará con 6 de sus 7 unidades, ocasionando que se deba disminuir la velocidad de la máquina para que la etiqueta dure más tiempo en cada una de las unidades. El tiempo de compra de los repuestos es de aproximadamente 5 a 21 días, dependiendo del tipo de repuesto, lo cual ocasiona que al no tener en existencias repuestos correspondientes a la parte dañada, esta bomba quedará detenida en su proceso productivo durante este tiempo.

Es de alta importancia el análisis de los compontes de falla de los elementos de las bombas, haciendo énfasis en el historial a corto plazo de una de estas bombas con las mismas características, debido a que con la información que se obtenga se podrán generar alternativas para mejorar la gestión de los inventarios de repuestos, y en consecuencia, el proceso de producción mediante la disposición de las bombas y su correcto funcionamiento en el proceso.

Con la técnica Cadenas de Markov se podrán modelar analizar los requerimientos de fallas de estas bombas, determinando así las probabilidades de ocurrencia al siguiente paso para determi- nar, en el tiempo, si las partes a reemplazar estarían disponibles según el stock y determinar también cuáles son los componentes que tienden a dañarse con más probabilidad de ocurrencia para tomar las acciones necesarias.

\section{Problema de investigación}

El sistema productivo de análisis ilustra un proceso de fabricación, desde el PVC en rollos, hasta el producto terminado que son las etiquetas termo-encogibles, bolsas de empaque al vacío, película de termo-formado y etiquetas wrap-around. Para este proceso la compañía tiene a su disposición equipos de producción; que tratan directamente con el proceso de trasformación, y equipos de apoyo que ayudan al proceso pero no están directamente relacionados con la transformación. Los equipos de apoyo en producción son: bombas peristálticas y gatos hidráulicos. Las bombas peristálticas son bombas hidráulicas de desplazamiento positivo, que son usadas para bombear fluidos, en este caso las tintas a las unidades de las máquinas markandys y la SMP, cuyo costo es de aproximadamente $500 \mathrm{USD} \mathrm{c} / \mathrm{u}$.

Su mantenimiento no es tan complejo, ya que consiste en el cambio de piezas específicas, cada vez que la bomba presenta algún problema por el desgaste de éstas, pero debido al repetitivo desgaste que presentan dichas partes, se elevan los costos de mantenimiento por el cambio de elementos a corto plazo y el tiempo del mecánico al cambiar las partes.

Se busca modelar el comportamiento actual de los requerimientos de estas bombas mediante una Cadenas de Markov, para confrontar este modelo con la política de inventario de estos repuestos, debido a que la política actual no está acorde con los tiempos de requerimientos, y al no contar con la existencia de alguno de los 6 repuestos disponibles es stock, desencadena los siguientes problemas:

Tiempo de bomba parada: cuando no se encuentra el repuesto que debe ser cambiado, la bomba queda en espera hasta que se obtenga el repuesto, teniendo en cuenta que los repuestos importados tienen un tiempo de entrega de 21 días, y los nacionales de 5 días como máximo. 
Unidad de la impresora SMP parada: cuando una bomba peristáltica no se encuentra en el proceso de bombeo de fluido de tinta a la unidad correspondiente, esta unidad no se puede utilizar, lo que ocasiona que se deba disminuir la velocidad de la impresora, según los parámetros de la máquina.

Productividad: al disminuir la velocidad de la máquina SMP, aumentan los tiempos para terminar un pedido, lo que afecta la productividad del proceso.

Costos: al no tener un repuesto en existencia del stock, y requerirlo con urgencia, se debe realizar un proceso de compra con envío rápido (repuestos importados), para que el tiempo de entrega disminuya de 21 días a 11 días, con costos adicionales por envío del $30 \%$.

Cuellos de botella: al tener un tiempo mayor en el proceso de impresión, los rollos de pvc salidos de refilarse por la máquina sliter_02, se acumulan en el proceso de impresión, lo que ocupa un espacio específico por más tiempo.

Por las anteriores razones, surge la importancia de analizar el modelamiento que se genere de dicho contexto, según las ventajas de Cadenas de Markov. Para ello se asocia la siguiente pregunta de investigación:

¿Cuál es el comportamiento del mantenimiento correctivo, según sus tipos de causas, en la bomba peristáltica, según probabilidades y tiempos promedio de ocurrencia?

\section{Objetivos}

\section{General}

Conocer el comportamiento de cambio de repuestos en las bombas peristálticas, para determinar el stock adecuado por medio de Cadenas de Makov.

\section{Específicos}

- Determinar las probabilidades de transición de un paso, con la información de los requerimientos de producción para mantenimiento correctivos de las bombas en la actualidad.
- Analizar el comportamiento de la estabilidad del sistema, conociendo sus probabilidades de ocurrencia para cada tipo de falla, y sus tiempos medios de primer paso y recurrencia.

- Comparar el comportamiento arrojado por Cadenas de Markov, con la política de inventario actual.

Las Cadenas de Markov son una herramienta de investigación de operaciones que permite identificar el comportamiento de una variable aleatoria según su estado actual, teniendo las probabilidades de tiempo estable y los tiempos medio de ocurrencia, lo que aplicado a este trabajo permitirá conocer, con los datos y condiciones actuales en las que se encuentran estas bombas (tiempo, operador, funcionamiento, tiempo), las probabilidades de que falle un repuesto al siguiente paso, teniendo presente que todas las partes de las bombas guardan interrelación entre ellas, y su vida útil depende del correcto funcionamiento de todas sus partes y su condición actual.

\section{MARCO TEÓRICO}

Un proceso estocástico se define como una colección indexada de variables aleatorias $\{\mathrm{Xt}\}$ donde el índice $t$ toma valores de un conjunto $T$ dado [3]. Existen diferentes técnicas de modelación alrededor de los procesos estocásticos, pero aquella que mejor representa un conjunto de variables aleatorias, en cuanto al análisis de su comportamiento, y teniendo en cuenta que dicho análisis no depende de la historia o del pasado registrado en su mismo comportamiento, lo ofrecen las Cadenas de Markov.

Una Cadena de Markov corresponde a una clase específica de proceso estocástico en el ámbito de modelos probabilísticos [2,8].

Se dice que un proceso estocástico tiene la propiedad markoviana si la probabilidad condicional de cualquier evento futuro dados cualquier evento pasado y el estado actual $X_{t}=i$, es independiente de los eventos pasados y sólo depende del estado actual del proceso [3].

El modelamiento markoviano está compuesto de la variable aleatoria, los estados de la naturale- 
za de interés y las probabilidades de transición entre ellos. Los acontecimientos se modelizan como pasos o transiciones de un estado a otros que se producen en periodos uniformes de tiempo, y con una probabilidad de transición que depende del estado en el que se encuentre el individuo en cada momento $[1,4,5]$.

Una herramienta fundamental para el estudio de las Cadenas de Markov lo constituyen las matrices de probabilidades de transición en $\mathrm{n}$ pasos: $P^{n}=P_{i j}^{n}$, donde $\mathrm{P}$ denota la probabilidad de que el proceso pase del estado $i$ al $j$ en $n$ pasos (tiempo).

$$
P_{i j}^{n}=P\left(X_{n+m}=j \mid X_{m}=i\right)
$$

Debe tenerse en cuenta algunos supuestos que según [5] son útiles para identificar la importancia y propósito del modelamiento desde las Cadenas de Markov:

- Solo se permiten unas transiciones entre estados previamente establecidas (finitas).

- La duración de los ciclos de Markov es arbitraria, debe ser constante a lo largo del estudio.

- La variable aleatoria está sometida a las mismas probabilidades de transición.

El análisis de los resultados obtenidos en la aplicación de la técnica obedece a dos aspectos: tiempo promedio y probabilidades de ocurrencia en el largo plazo. La literatura más reciente, según [6], relacionada con el análisis de datos y su interacción con las Cadenas de Markov se centra principalmente en el cálculo del tiempo promedio en que la variable aleatoria puede transitar en diferentes estados de interés.

Las probabilidades de estado estable indican aquella proporción de encontrar el proceso en determinado estado, por ejemplo j, después de un número grande de transiciones tiende al valor $\pi_{\mathrm{j}} \mathrm{y}$ es independiente de la distribución de probabilidad inicial definida para los estados [3].

Otro análisis interesante, es el estudio del tiempo que el sistema permanece en el estado i antes de abandonarlo. Esto es, estudiar el tiempo promedio de permanencia entre diferentes estados [7].

\section{Contexto de Desarrollo}

El contexto donde se desarrollada este estudio corresponde al área de mantenimiento, el cual trata de la manutención y control de los equipos que hacen parte de un proceso productivo con el objeto de alargar su vida útil.

Las variables y elementos que intervienen en el desarrollo de este informe son los siguientes:

\section{Identificación de estados y probabilidades de transición}

La variable aleatoria de interés obedece al mantenimiento correctivo que se debe realizar debido a las fallas que presenta la bomba en el proceso de producción, cada vez que ocurre un requerimiento de producción, los encargados de planta deben analizar si se presentará o no fallo de dicha bomba, y en caso de presentarse, estudiar a profundidad el tipo de falla.

\section{$X_{t}$ : Mantenimiento corrrectivo de las bombas en el requerimiento de producción $t$}

Los estados se asocian con las posibles causas de mantenimiento correctivo, que pueden estar entre:

Elementos: son aquellos repuestos identificados de las bombas peristálticas.

Piñones L112Y: Boston Gear L112Y Mitre engranaje, 1: 1, ángulo de presión de 20 grado, 0.500 «de diámetro, 1.375» Distancia de montaje, 16 Pitch, 24 dientes, de acero. Straight inglete 90 de malla de grado transmite la potencia mecánica entre ejes perpendiculares. Ángulo de presión de 20 grados para una relación de contacto inferior entre los engranajes, lo que resulta en una capacidad de carga superior a ángulos de presión más pequeñas. Acero bajo en carbono $(0,2$ por ciento de carbono) para aplicaciones en las que la dureza y la fuerza de los niveles más altos de carbono.

Procedencia: importada y nacional.

Escobillas: es un Cepillo de carbono 15,7 x 9,9 $\mathrm{x}$ $5,9 \mathrm{~mm} / 8.5 \ll x 25 / 64 » x 15 / 64 \ll\left(\mathrm{L}^{*} \mathrm{~W}\right.$ * $\left.\mathrm{T}\right)$, Longitud del cable: $20 \mathrm{~mm} /$ 0,8»; Material: Carbono, Metal. Procedencia: importada. 
Rotor: elemento de transferencia mecánica, ya que de él depende la conversión de energía eléctrica a mecánica. Procedencia: importada.

Motor: modelo N6565 Categoría 33A-Z eje paralelo DC Motorreductor Velocidad (rpm) 417 Par de giro nominal (lb-in) 10 Tensión nominal de 130V amperios (24V modelo) 4 amperios (130V modelo) 0,71 Par máximo (lb-in) $13 \mathrm{CV}$ del motor 1 / 12 Relación de engranajes 6 carga radial (100 libras) Longitud XH (pulgadas) Peso (7 libras). Procedencia: importada

Eje: pieza donde se apoya el rodamiento para lograr que gire al sentido del motor. Procedencia: nacional.

Rodamiento R8ZZ: Rodamientos rígidos de bolas, diámetro interior (d): $12.7 \mathrm{~mm}$, diámetro exterior (D): $28.575 \mathrm{~mm}$, ancho(B): $7.938 \mathrm{~mm}, \mathrm{Cr}: 5216.4$ N, C0r: 2440.368 N, RPM Aceite: 30000 1/min, peso(m): 0.0227 k, tipo de sello: metálico. Procedencia: nacional.

Se determinan las probabilidades de transición mediante el registro diario de los fallos que se presentan en la planta (solo relacionados con el proceso productivo, no se incluyen reparaciones de infraestructura), el cual se obtiene mediante un formato llamado «requerimiento de trabajo», donde se describe el problema y la solución. Para la consecución de las probabilidades de transición se filtraron los requerimientos en la bomba \#1 de 24 observaciones tomadas en 1 mes obteniendo la siguiente información:

- Cuando fallaron en 4 ocasiones los piñones de la bomba N.1 se tuvo que cambiar una vez el rotor, otra vez el motor, y a la tercera falla de haberse cambiado el otro piñón, se cambió el eje, y la cuarta vez que fallo un piñón, de nuevo se tuvo que cambiar otro piñón .

- Cuando fallaron en 2 ocasiones las escobillas, se tuvieron que cambiar en el siguiente requerimiento un Piñón, y después de la segunda falla de una escobilla, se tuvo que cambiar un rotor.

- Cuando falló en 1 ocasión el rotor, se tuvo que cambiar a la siguiente vez las escobillas.

- Cuando falló en 1 ocasión el motor, se tuvo que cambiar a la siguiente vez un Piñón.

- Cuando fallaron en 2 ocasiones los ejes, se presentó un cambio de una vez un piñón, y a la segunda falla se presentó un cambio para el otro eje, en el siguiente requerimiento se tuvo que cambiar 1 rodamiento.

- Cuando falló un rodamiento al siguiente requerimiento se tuvo que cambiar un eje.

\section{Observaciones}

De la anterior recolección de datos se tomó 1 bomba peristáltica, de la máquina SMP.

Las 7 bombas tienen las mismas características, todas tienen 3 años, son del mismo fabricante, trabajan las mismas horas al día, y las operan las mismas personas.

A continuación se ilustra la matriz de probabilidades de transición obtenidas según el análisis anterior (tabla 1).

Tabla 1. Matriz de probabilidades de transición

\begin{tabular}{lccccc}
\hline $\begin{array}{l}\text { Matriz de probabilidades } \\
\text { de transición }\end{array}$ & $\begin{array}{c}\text { Piñones } \\
\text { L112Y }\end{array}$ & Escobillas & Rotor & Motor & $\begin{array}{c}\text { Ejes } \\
\text { Rodamiento } \\
\text { R8ZZ }\end{array}$ \\
\hline Piñones L112Y & 0,25 & 0 & 0,25 & 0,25 & 0,25 \\
Escobillas & 0,5 & 0 & 0,5 & 0 & 0 \\
Rotor & 0 & 1 & 0 & 0 & 0 \\
Motor & 1 & 0 & 0 & 0 & 0 \\
Ejes & 0,5 & 0 & 0 & 0 & 0 \\
Rodamiento R8ZZ & 0 & 0 & 0 & 0 & 0 \\
\hline
\end{tabular}

Fuente: los autores. 
Obteniendo como resultado el siguiente diagrama, que describe la situación del mantenimiento correctivo, como una Cadena de Markov (Fig. 1).

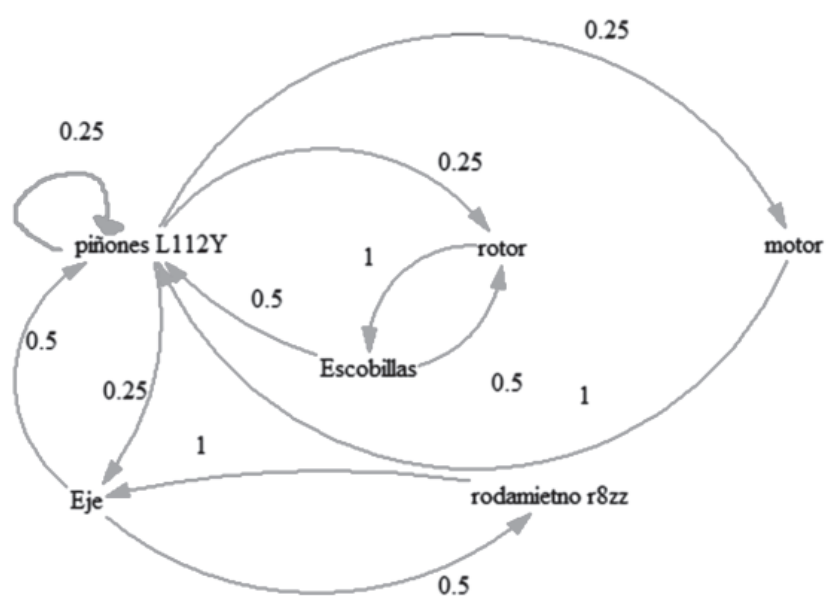

Fig. 1. Grafo de la cadena de Markov del mantenimiento correctivo. Fuente: los autores.

\section{Estabilidad del sistema}

Las probabilidades de estado estable calculadas indican que (tabla 2).

A la larga a través de tiempo la probabilidad de que los requerimientos sean para cambio de piñones L112Y va hacer del 33.33\%, sin importar los estados anteriores. El cambio de escobillas o rotor se presentará un $16.67 \%$ de las veces. El cambio de motor se presentará en una proporción de $8.33 \%$. Cambio de ejes corresponderá a un $16.67 \%$. Y cambio de rodamientos se presentará un $8.34 \%$ de las veces.

Tiempos medios de recurrencia y de primer paso (tablas 3 y 4 )

M11= 3 En promedio pasaran aproximadamente 3 requerimientos de producción para que se requiera cambiar de nuevo un piñón L112Y.

Tabla 2. Probabilidad de estado estable.

\begin{tabular}{|c|c|c|c|c|c|c|}
\hline $\begin{array}{l}\text { Probabilidades } \\
\text { de estado estable }\end{array}$ & $\begin{array}{l}\text { Piñones } \\
\text { L112Y }\end{array}$ & Escobillas & Rotor & Motor & Ejes & $\begin{array}{c}\text { Rodamiento } \\
\text { R8ZZ }\end{array}$ \\
\hline Piñones L112Y & 0,3333 & 0,1667 & 0,1667 & 0,0833 & 0,1667 & 0,0833 \\
\hline Escobillas & 0,3333 & 0,1667 & 0,1667 & 0,0833 & 0,1667 & 0,0833 \\
\hline Rotor & 0,3333 & 0,1667 & 0,1667 & 0,0833 & 0,1666 & 0,0833 \\
\hline Motor & 0,3333 & 0,1667 & 0,1667 & 0,0833 & 0,1667 & 0,0833 \\
\hline Ejes & 0,3333 & 0,1666 & 0,1667 & 0,0833 & 0,1667 & 0,0833 \\
\hline Rodamiento R8ZZ & 0,33333 & 0,1667 & 0,1666 & 0,0833 & 0,1666 & 0,0834 \\
\hline
\end{tabular}

Fuente: los autores.

Tabla 3. Tiempos promedio de recurrencia.

\begin{tabular}{|c|c|c|c|c|c|c|}
\hline Tiempos medios de recurrencia & $\begin{array}{c}\text { Piñones } \\
\text { L112Y }\end{array}$ & Escobillas & Rotor & Motor & Ejes & $\begin{array}{c}\text { Rodamiento } \\
\text { R8ZZ }\end{array}$ \\
\hline Requerimiento de producción & 3,00 & 6,00 & 6,00 & 12,00 & 6,00 & 12,00 \\
\hline
\end{tabular}

Fuente: los autores.

Tabla 4. Tiempos promedio de primer paso y recurrencia.

\begin{tabular}{lccccc}
\hline $\begin{array}{l}\text { Tiempos promedio de primer paso y recurrencia } \\
\text { Rodamiento R8ZZ }\end{array}$ & Piñones L112Y & Escobillas & Rotor & Motor & Ejes \\
\hline Piñones L112Y & 3,00 & 1,33 & 1,33 & 1,33 & 1,33 \\
Escobillas & 0,00 & 6,00 & 1,66 & 1,66 & 1,66 \\
Rotor & 1,00 & 0,00 & 6,00 & 0,00 & 0,00 \\
Motor & 0,00 & 0,00 & 2,33 & 12,00 & 2,33 \\
Ejes & 0,00 & 0,00 & 1,66 & 1,66 & 6,00 \\
Rodamiento R8ZZ & 1,00 & 0,00 & 0,00 & 0,00 & 0,00 \\
\hline
\end{tabular}

Fuente: los autores. 
M22= 6 En promedio pasaran aproximadamente 6 requerimientos de producción para que se requiera cambiar de nuevo una escobilla.

M33= 6 En promedio pasaran aproximadamente 6 requerimientos de producción para que se requiera cambiar de nuevo un rotor.

M44=13 En promedio pasaran aproximadamente 13 requerimientos de producción para que se requiera cambiar de nuevo un motor.

M55= 6 En promedio pasaran aproximadamente 6 requerimientos de producción para que se requiera cambiar de nuevo un eje.

M66= 13 En promedio pasaran aproximadamente 13 requerimientos de producción para que se requiera cambiar de nuevo un rodamiento R8ZZ.

Se logra identificar en la tabla anterior el tiempo promedio (en términos de los requerimientos de producción) que pasará el escenario de la falla de la bomba en cada tipo de causa, antes de presentar una falla en el futuro frente a otro tipo. Esto es, por ejemplo:

Identificándose inicialmente un requerimiento para cambio de un piñón L112y, pasarán en promedio 2 requerimientos de producción antes de requerir un cambio escobillas, de rotor, de motor o de ejes.

Identificándose un requerimiento para cambio de un piñón L112y, pasará en promedio 1 requerimiento de producción antes de requerir un cambio de rodamiento R8ZZ.

Identificándose inicialmente un requerimiento para cambio de escobillas, no pasara a un requerimiento para cambio de un piñón L112Y, ni para cambio de rodamiento R8ZZ. Pero si se pronostica un paso de 2 requerimientos en promedio para presentar cambio de rotor, motor y ejes.

\section{Resultados}

El piñón L112Y presenta mayor frecuencia de solicitudes de cambio alrededor del tiempo, lo que implica establecer una política de gestión de inventarios con mayor nivel de rotación o frecuencia de análisis y control.

Escobillas, rotor y ejes son partes de la bomba que presentarán un 16,67\% de solicitudes de cambio en el tiempo. No presentan una necesidad de rotación alta en sus inventarios, pero si es importante tener en cuenta esta frecuencia de ocurrencia, para determinar políticas de gestión de inventarios alrededor del tema.

Motor y rodamiento son las partes que menos solicitudes de cambio requerirán en el tiempo. Un $8 \%$ de las veces en que se requiera mantenimiento correctivo, acaecerá una relación directa hacia un cambio en este tipo de partes.

En definitiva, debe replantearse la política de gestión del inventario de estos repuestos, dado que no ha sido suficiente para suplir la demanda interna, para reemplazo de los repuestos, lo que ocasiona demoras en el proceso.

Se debe tener en cuenta para el replanteamiento del nivel del inventario adecuado, lo siguiente; si cada 3 requerimientos de producción en promedio se necesitará un Piñón se deberá tener como mínimo 4 piñones mensuales en inventario, también si necesitara cada 6 requerimientos en promedio cambio de escobillas, se deberá tener como mínimo 2 escobillas en el mes, lo mismo pasará con el rotor y los ejes, y si conozco que en promedio cada 13 requerimientos se necesitará de nuevo un motor o un rodamiento, deberá tener como mínimo 1 repuestos de cada uno. Esto para garantizar desde el punto de vista de Cadenas de Markov aplicada a este problema que los repuestos siempre estará disponible (tabla 5).

Si se tiene en cuenta el escenario estudiado mediante la Cadena de Markov asociada al contexto del control de los repuestos, se evidencia que se deben cambiar las políticas de gestión del inventario actuales para los piñones, escobillas y rotores. Actualmente se presenta un faltante del $50 \%$ frente a lo pronosticado.

Si se continúa con la gestión actual del stock para estas partes, puede incurrirse en faltantes, tiempos de demora en la entrega de las mismas, y en definitiva, ocasionaría los paros en el proceso productivo, asociados a la misma causa. 
Tabla 5. Comparativo políticas de gestión de inventarios.

\begin{tabular}{lccc}
\hline Requerimiento de stock & Stock mínimo (deseado) & Stock mínimo (actual) & DIFF \\
\hline Piñones L112Y & 4 & 2 & $50 \%$ \\
Escobillas & 2 & 1 & $50 \%$ \\
Rotor & 2 & 1 & $50 \%$ \\
Motor & 1 & 1 & $0 \%$ \\
Ejes & 2 & 4 & N/A \\
Rodamiento R8ZZ & 1 & 2 & N/A \\
\hline
\end{tabular}

Fuente: Los autores

\section{CONCLUSIONES}

Las Cadenas de Markov permiten conocer el comportamiento de un modelo que cumpla la característica de que un evento depende de otro inmediatamente anterior, con lo cual se estudian diferentes situaciones a nivel industrial y de la vida diaria como es el hecho de determinar que un sistema con partes interrelacionadas tengan dependencia una con las otras y así con todas las demás partes, y esto se comprueba con las especificaciones en este caso del manual del fabricante.

También ayuda a determinar tomando como base este modelo, que en la gestión de un sistema de inventario de repuestos debe tenerse en cuenta las situaciones actuales en las que se relacionan los equipos, debido a que estás tienen a influir en un futuro corto, como es en este caso, la persona que opera las bombas, tiempo de trabajo y vida útil de las mismas.

Si conocemos la probabilidad de que un repuesto pase al siguiente paso, con el stock mínimo hallado con las probabilidades se podrá identificar disponibilidades, y en consecuencia, evitarán retrasos en el proceso productivo, debido a faltantes en los repuestos de la máquina de interés.

\section{RECOMENDACIONES}

Es importante que en el momento de realizar este tipo de estudios, a nivel productivo, se tenga acceso a la información preliminar para realizar cálculos con validez suficiente para determinar las probabilidades de transición.

\section{REFERENCIAS}

[1] A. Briggs, M. Sculpher. An introduction to Markov modelling for economic evaluation. Pharmacoeconomics 1998;13:397-409.

[2] D.P. Bertsekas, J.N. Tsitsiklis. «Introduction to Probability». Athena Scientific. USA. 2002.

[3] F. Hillier, Introducción a la Investigación de Operaciones. Novena edición. McGraw Hill. México, 2010.

[4] J. Latour. Análisis de decisiones. Quaderns de salut pública y administració de serveis de salut, 12. Valencia, 1997.

[5] J.R. Beck, S.G. Pauker. The Markov Process in medical prognosis. Med Decision Making 1983;3(4):19-58.

[6] J. Valdés. Aporte de las Cadenas de Markov al análisis de la tasa de desempleo de largo plazo. Estado y evolución para la república Argentina. 2006.

[7] L. Gorno. Cadenas de Markov Homogéneas. Facultad de Ciencias Económicas. Universidad de Buenos Aires, Argentina, 2003.

[8] S. Ross. «Introduction to Probability Models». Academic Press. USA. 2000. 